\title{
A Rare but Potentially Fatal Combination of Emphysematous Liver Abscess and Renal Cortical Abscess by Proteus Mirabilis in a Critically Ill Diabetic Patient
}

\begin{abstract}
Jayaraman $\mathrm{R}^{1 *}$, Aravindh $\mathrm{G}^{1}$, Banoo $\mathrm{S}^{2}$, Naveen $\mathrm{K}^{3}$, Kumar $\mathrm{A}^{4}$, Bhalaji $\mathrm{S}^{4}$, Lakshmi $\mathrm{N}^{5}$, Karthikeyan $\mathrm{K}^{6}$, Cheran $\mathrm{I}^{6}$ and Kumar $\mathrm{S}^{7}$

${ }^{1}$ Consultant nephrologist, Dhanalakshmi Srinivasan Medical College and Hospital, India

${ }^{2}$ Additional professor in microbiology, Dhanalakshmi Srinivasan Medical College and Hospital, India
\end{abstract}

${ }^{3}$ Assistant professor of radiology, Dhanalakshmi Srinivasan Medical College and Hospital,

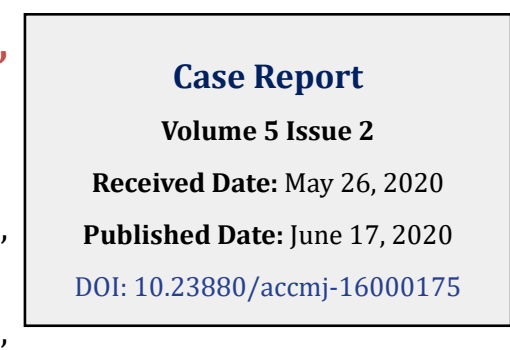
India

${ }^{4}$ Consultant urologist, Dhanalakshmi Srinivasan Medical College and Hospital, India

${ }^{5}$ Department of microbiology, Dhanalakshmi Srinivasan Medical College and Hospital, India

${ }^{6}$ Department of radiology, Dhanalakshmi Srinivasan Medical College and Hospital, India

${ }^{7}$ Department of nephrology, Dhanalakshmi Srinivasan Medical College and Hospital, India

*Corresponding author: Rajesh Jayaraman, Consultant nephrologist, Dhanalakshmi Srinivasan Medical College and Hospital, Trichy - Chennai highway, Perambalur district, Tamilnadu, India, Tel: 82482 11454; Email: rajsubbuiyer@gmail.com

\section{Abstract}

Emphysematous liver abscess is a rare and distinct entity that occurs due to fulminant infection of the liver by gas forming organisms. It most commonly occurs in patients with uncontrolled diabetes mellitus akin to emphysematous pyelonephritis that can have a rapid and downhill course. We hereby report a case of middle-aged diabetic woman with Proteus Mirabilis induced emphysematous liver abscess complicated with renal stone disease and left renal abscess with probable kidney to liver spread as the cause.

Keywords: Emphysematous Liver Abscess; Renal Cortical Abscess; Proteus Mirabilis

\section{Introduction}

While emphysematous pyelonephritis is a well-known entity in the domain of nephrology, emphysematous hepatic suppuration is not as common as the former. E.coli, Klebsiella pneumoniae and Proteus species are the main source of gas production in such cases that almost invariably occurs in patients with poorly controlled diabetes mellitus and can have a rapidly downhill course. In our case, we had a patient simultaneous liver and renal suppuration by Proteus Mirabilis surprisingly with gas formation in liver alone. Liver abscess was percutaneously drained with the typical gas evolution. Renal stone obstruction was managed through DJ stenting. Patient did not agree for renal abscess drainage though. The patient finally succumbed to various comorbidities. The peculiarity in our case is the emphysematous nature of the liver abscess which is not that common as that of emphysematous pyelonephritis and the probable renohepatic spread of the infection as suggested by the cultured organism in both the liver abscess and the urine of the patient, Proteus Mirabilis. 


\section{Case report}

This 48-year-old female, a diabetic of over 12 years and poorly controlled got admitted six months ago in our hospital with two weeks history of high-grade fever, diffuse abdominal pain, several episodes of vomiting, decrease in urine output followed by obtundation. On examination, she was febrile, drowsy and dehydrated. Her blood pressure was $90 / 60 \mathrm{~mm}$ of $\mathrm{Hg}$ requiring inotropic support. Her blood reports revealed advanced azotemia necessitating dialysis. Her WBC counts were elevated and her coagulation profile was grossly deranged. Her biochemistry was as follows: CBC - 21,000, Hb-7.1gm\%, PT, APTT, INR- deranged. Serum creatinine was $6.8 \mathrm{mg} \%$, Total bilirubin $-3.3 \mathrm{mg} \%$, SGOT, SGPT $>200 \mathrm{IU} / \mathrm{L}$, serum procalcitonin was also elevated. Her blood sugar levels were above $400 \mathrm{mg} \%$ and $\mathrm{HbA} 1 \mathrm{C}$ was $11.2 \%$. Urine showed numerous pus cells on examination.

Her contrast CT abdomen/KUB revealed 10.1x $9 \mathrm{~cm}$ peripherally enhancing hypodense lesion in the right lobe of the liver with gas shadows within, consistent with emphysematous liver abscess. Her left kidney showed pyelonephritic changes including perinephric fat stranding along with an abscess of size $4.3 \mathrm{~cm}$ in its lower pole, though obvious gas shadows suggestive of emphysematous pyelonephritis was absent. The patient also had stones in both kidneys and ureter with hydronephrosis. There was no evidence of renal papillary necrosis.

Insulin was employed for glycemic control. Liver abscess was aspirated percutaneously under CT guidance and around $160 \mathrm{ml}$ of thick toffee colored pus was aspirated with typical gas evolution. Patient was immediately started on Meropenam to have a good coverage for pseudomonas also (Figures 1-5).

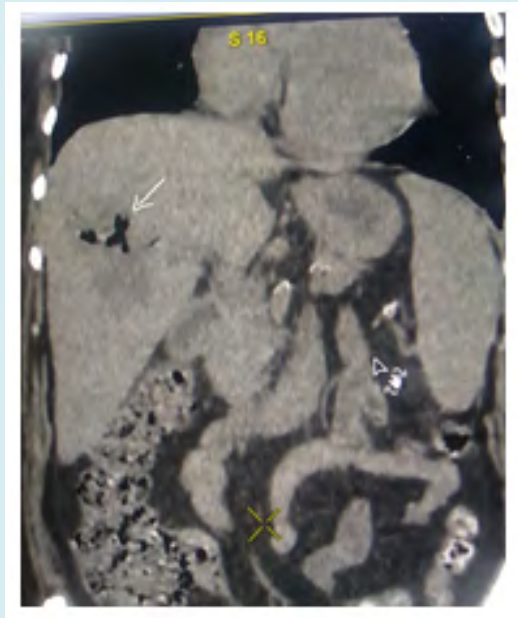

Figure 1: Arrow showing the emphysematous suppuration of the liver.

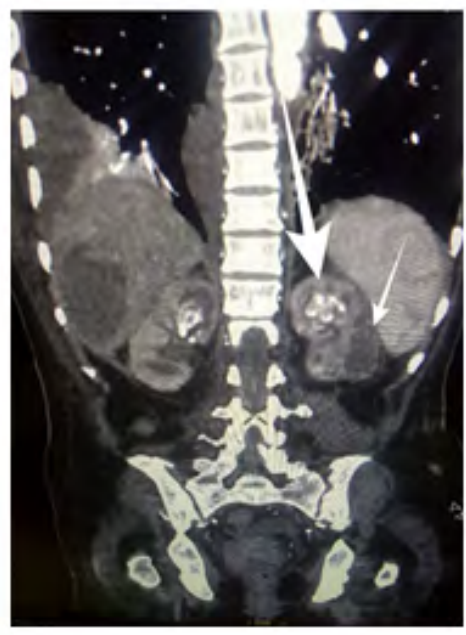

Figure 2: The left renal cortical abscess in the lower pole (smaller arrow) along with the multiple stones (the bigger arrow).

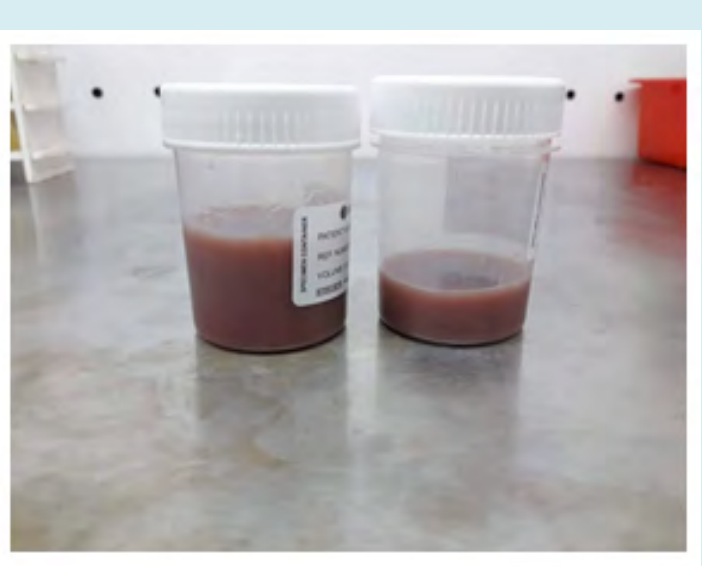

Figure 3: The toffee coloured pus aspirated from liver.

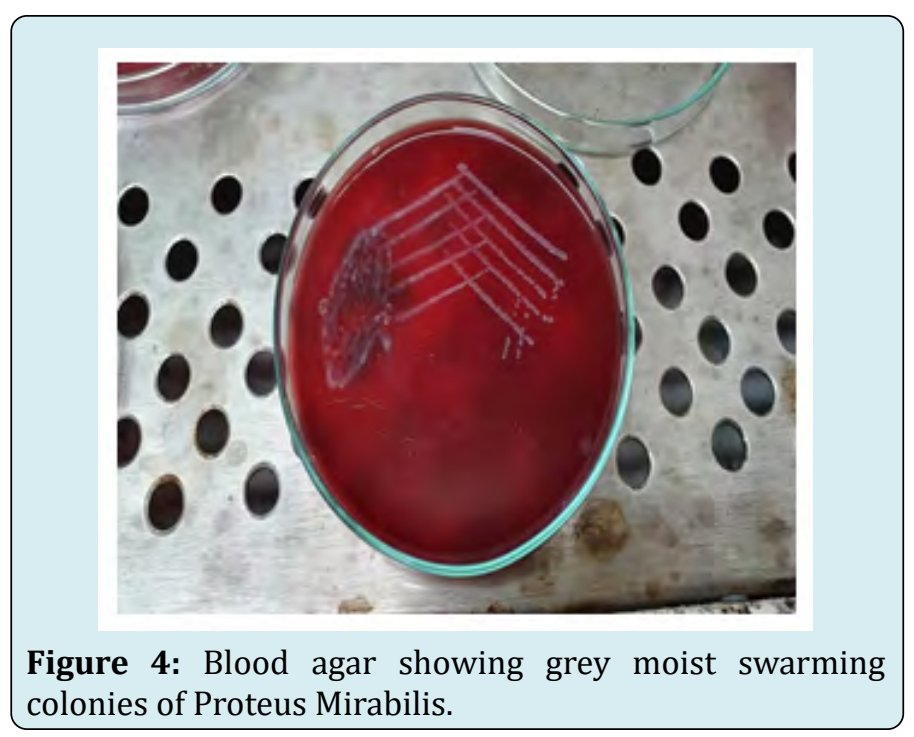




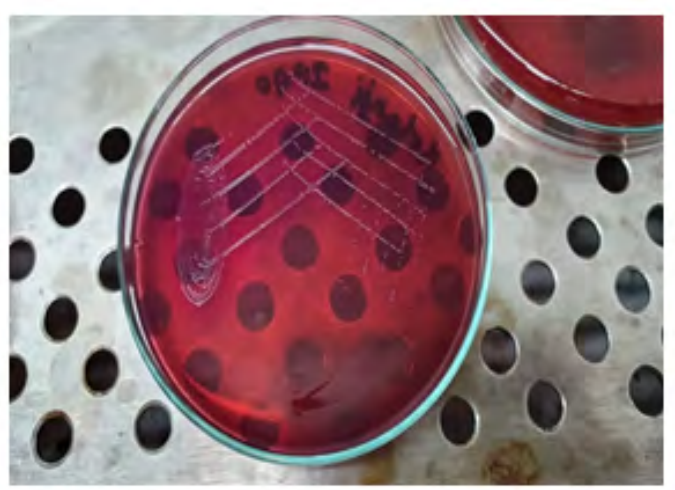

Figure 5: Macconkey agar showing pale non-lactose fermenting colonies.

Pus sample thus aspirated was sent for aerobic and anaerobic culture and sensitivity along with a simultaneously collected urine sample for culture. Gram staining of pus showed plenty of pus cells along with gram negative, pleomorphic, non-capsulated rods. Sample was streaked in blood and MacConkey agar. Both aerobic and anaerobic culture in blood agar showed non-hemolytic, grey, moist colony with swarming growth and fishy odor revealing Proteus Mirabilis colonies of more than $10^{5}$ colony forming units / $\mathrm{ml}$. MacConkey agar showed pale non-lactose fermenting colonies. Biochemical reactions were consistent with Proteus Mirabilis. For both urine and pus samples, antibiotic susceptibility test was performed based on CLSI (Clinical and Laboratory Standards Institute) 2019 guidelines. Both the samples showed similar pattern of sensitivity to cephalosporins, tigecycline, carbapenams and resistant to fluoroquinolones, aminoglycosides, cotrimoxazole and nitrofurantoin group of drugs. Meropenam was hence continued along with inotropic and dialysis support. Bilateral DJ stenting was done for decompressing the obstructed urinary tract with proper stone retrieval postponed to a later date. Patient was also informed about the need of surgical drainage of the renal abscess for which the attenders did not agree.

Though initially patient showed a modest improvement in the form of increasing urine output and decreasing total leucocyte count, her condition gradually worsened over subsequent days. She also developed severe bleeding diathesis probably secondary to sepsis and disseminated intravascular coagulation. Despite the aggressive management of sepsis and renal failure, the patient succumbed to the illness.

\section{Discussion}

Emphysematous liver abscess accounts for $6.6-24 \%$ of all cases of liver abscess [1-4]. While it occurs almost always in patients with poorly controlled diabetes mellitus, the mortality associated with emphysematous liver abscess can be as high as 30\% necessitating prompt intervention and management. Chou FF and Sheen-Chen SM [5] reported an overall mortality of $27.7 \%$ in emphysematous liver abscess cases over a mortality of $14.4 \%$ in non- emphysematous cases.

The mechanism of gas formation is often postulated to be directly linked with the poorly controlled glycaemic status. Hsin-Ling Lee and Hsin-Chun Lee [3] in their study on 22 patients of emphysematous liver abscesses, could establish mixed acid fermentation of glucose as the reason for the net gas production. They hypothesized that the gas formation involves increased production of gas, impaired transportation of gas, and equilibrium between the gas in local tissues and that in abscesses. As nitrogen is ubiquitous in all body fluids and tissues in high concentrations, it is not surprising that nitrogen levels were high in gas samples taken from abscesses.

The microbiology of gas forming infection and suppuration of any solid viscus is of a wider spectrum and could be polymicrobial. The most common organisms implicated are E.Coli, Pseudomonas Aeroginosa, Klebsiella Pneumoniae and Proteus Mirabilis [6]. Coeway Boulder Thng, et al. [7] in their recent study found out that 30\% infections in pyogenic liver abscess as due to gas forming organisms and that, Klebsiella Pneumoniae as the most common gas forming organism. In the same study, mortality was $30.3 \%$ in pyogenic liver abscess due to gas forming organisms versus $9 \%$ in non-gas forming organisms. In our case, both urine and liver abscess culture yielded Proteus Mirabilis with the same sensitivity in antibiogram. Blood culture was negative in our patient.

There are no available adequate case reports of haematogenous spread of emphysematous hepatic suppuration to the kidneys or vice versa. Ho Youn Lee [8] reported a case of septic pulmonary embolism due to pyelonephritis. We weren't able to trace any case report citing the clear haematogenous spread of hepatic emphysematous abscess to the kidneys or vice versa. In our case, though the liver abscess was percutaneously drained, we were not able to do surgical drainage of the renal abscess, as the patient did not give consent.

Most of the times in a diabetic patient, occurrence of pyelonephritis is associated with renal stone disease/ obstruction coupled with uncontrolled diabetes. Ubee SS and McGlynn L [9] quoted a risk of an additional 25$40 \%$ for developing emphysematous pyelonephritis in a diabetic patient with obstructive renal stones. Tanmaya Goel and Sreedhar Reddy [10] have reported 7 cases of 


\section{Anaesthesia \& Critical Care Medicine Journal}

emphysematous pyelonephritis, out of which 4 had urinary tract obstruction secondary to renal stones. Following the initial conservative management by DJ stenting and PCN, they successfully did stone retrieval over the follow-up. In our case, both the kidneys had stones, $2.5 \mathrm{~cm}$ in size in the upper pole of each kidney along with a $14.5 \mathrm{~mm}$ lower ureteric calculus on the right side. We managed the obstructions with DJ stenting on both the sides and had planned for the actual stone management at a later date.

Renal cortical and corticomedullary abscesses/perirenal abscesses occur in less than 10 of 10000 hospital admissions [11]. A pure renal cortical abscess, also referred to as renal carbuncle is usually the result of haematogenous spread from elsewhere and staphylococcus aureus is the usual pathogen. Treatment with antibiotics shall usually be effective in such cases. A renal corticomedullary abscess, in contrast, usually results from ascending urinary tract infection in association with an underlying urinary tract abnormality, such as obstructive uropathy. The pathogens here are the usual uropathogenic species such as E. coli and other coliforms [12].

Most of the renal corticomedullary abscesses require percutaneous or surgical drainage apart from broad spectrum antibiotic therapy. In our case, the abscess of size $4.3 \mathrm{~cm}$ in the lower pole of left kidney was purely cortical with perinephric extension and peculiarly, the microbiological flora was one of the typical uropathogens Proteus Mirabilis, unlike Staphylococcus aureus in the usual scenario. So, based on the isolated organism, we strongly suspect a possibility of reno-hepatic spread in our patient, though obvious gas formation was absent in the renal source of suppuration. To the best of our knowledge, there are no reported cases of Proteus species associated emphysematous liver abscess with renal abscess as the probable source.

\section{Conclusion}

Emphysematous liver abscess comprises approximately 6-24\% cases of all liver abscesses and carries a mortality of up to 30\%. E. Coli, Klebsiella Pneumoniae, Proteus and Pseudomonas are the responsible flora. Renal cortical abscess is usually the result of haematogenous spread from a distant organ and staphylococcus aureus is the usual culprit, while renal cortico- medullary abscess is usually a result of the ascending infection by the microbiological flora of the urinary tract, namely E. Coli, Klebsiella and Proteus. As we encountered Proteus Mirabilis as the cultured organism from both the sources of suppuration, we strongly consider a possibility of reno-hepatic spread of the infection in our case.

\section{References}

1. Hagiya H, Kuroe Y, Nojima H, Otani S, Sugiyama J, et al. (2013) Emphysematous liver abscesses complicated by septic pulmonary emboli in patients with diabetes: two cases. Intern Med 52(1): 141-145.

2. Kaplan GG, Gregson DB, Laupland KB (2004) Populationbased study of the epidemiology of and the risk factors for pyogenic liver abscess. Clin Gastroenterol Hepatol 2(11): 1032-1038.

3. Lee HL, Lee HC, Guo HR, Chien Ko W, Chen KW (2004) Clinical significance and mechanism of gas formation of pyogenic liver abscess due to Klebsiella pneumoniae. J Clin Microbiol 42(6): 2783-2785.

4. (1996) The Diabetes Control and Complications Trial (DCCT) Research Group The absence of a glycemic threshold for the development of long-term complications: the perspective of the Diabetes Control and Complications Trial. Diabetes 45(10): 1289-1298.

5. Chou FF, Sheen-Chen SM, Chen YS (1995) The comparison of clinical course and results of treatment between gas-forming and non-gas-forming pyogenic liver abscess. Arch Surg 130(4): 401-405.

6. McHugh TP, Albanna SE (1998) Bilateral emphysematous pyelonephritis. Am J Emerg Med 16(2): 166-169.

7. Coeway Boulder Thng, Yen Pin Tan, Shelat VG (2018) Gas-forming pyogenic liver abscess: A world review. Ann Hepatobiliary Pancreat Surg 22(1): 11-18.

8. Ho Youn Lee (2009) A case of septic pulmonary embolism due to pyelonephritis: The Korean Journal of Medicine 76(1): 105-109.

9. Ubee SS, McGlynn L (2011) Emphysematous pyelonephritis. BJU Int 107(9): 1474-1478.

10. Tanmaya Goel, Sreedhar Reddy, Joseph Thomas (2007) Emphysematous pyelonephritis with calculus: Management strategies. Indian J Urol 23(3): 250-252.

11. Dembry LM, Andriole VT (1997) Renal and perirenal abscesses. Infect Dis Clin North Am 11(3): 663-680.

12. Urinary tract infection in adults: Comprehensive clinical nephrology ( $6^{\text {th }}$ edn), chapter 51; page 634-635.

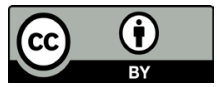

